\title{
Taken last, selected first: The sampling bias is also present in the haptic domain
}

\author{
Takashi Mitsuda • Yuichi Yoshioka
}

Published online: 3 December 2014

(C) The Psychonomic Society, Inc. 2014

\begin{abstract}
When people are presented with a pair of images and asked to identify which one is more attractive, their eye gaze shifts gradually toward the image that they eventually choose. This study examined whether this sampling bias also occurs in other sensory modalities by observing participants' behavior in a haptic preference task. The results indicated that the participants tended to sample the chosen item just prior to making their decision when they were instructed to identify their most preferred item (i.e., the "like" task), but not when they were instructed to identify their least preferred item (i.e., the "dislike" task). This indicates that the sampling bias is a general phenomenon regardless of sensory modality. In addition, the sampling bias in the like task was larger when the difference in preference ratings between the paired items was smaller. However, the sampling bias decreased when the two items were given equal preference ratings, despite there being a longer decision time on those trials. This suggests that the sampling bias is not simply related to task difficulty, but is also related to preference formation and/or selective encoding of task-relevant information.
\end{abstract}

Keywords Gaze bias · Preference · Haptic · Tactile sense · Decision making $\cdot$ Dislike

\section{Introduction}

Making choices between multiple items is a fundamental activity in daily life. For example, when selecting a blouse to purchase in a shop, people usually examine and compare the various candidates repeatedly. Shimojo, Simion, Shimojo,

T. Mitsuda $(\bowtie) \cdot$ Y. Yoshioka

College of Information Science and Engineering, Ritsumeikan University, 1-1-1 Noji-Higashi, Kusatsu, Shiga 525-8577, Japan

e-mail:mitsuda@ci.ritsumei.ac.jp and Scheier (2003) have shown that this looking behavior is related not merely to item evaluation, but also to preference formation. These latter authors tracked the eye movements of individuals who were shown pairs of human faces and asked to decide which face was more attractive. The results showed that participants' eye gazes were initially distributed evenly between the two stimuli, but approximately $1 \mathrm{~s}$ prior to making a decision, they gradually shifted their eyes toward the face they subsequently chose. However, this "gaze bias" was significantly weaker when the participants were asked to select the less attractive, or the rounder, of the two faces. As a result of this study, looking behavior in preference-judgment tasks has been well researched in the literature (Glaholt \& Reingold, 2009; Krajbich, Armel, \& Rangel, 2010; Mitsuda \& Glaholt, 2014; Nittono \& Wada, 2009; Schotter, Berry, McKenzie, \& Rayner, 2010). Although the source of the gaze bias is still being debated, there is evidence indicating that it is not a response-related phenomenon; rather, it may be related to the decision-making process.

Recently, Lindsen, Gurpreet, Shimojo, and Bhattacharya (2011) showed that the behavior of sampling musical excerpts was also biased when participants were asked to choose an excerpt that they liked, but not when asked to choose one they did not like; thus, the observed bias was similar to the gaze bias. These results indicate that these types of biases could be a general tendency in preference-judgment tasks, regardless of the sensory modality. To the best of our knowledge, Lindsen et al. (2011) is the only study that has investigated the sampling bias in preference-judgment tasks in sensory modalities other than vision. Accordingly, we tested whether the sampling bias also exists in the sensory domain of tactile processing.

The present study introduced a two-alternative forcedchoice task that asked participants to indicate their preference for handkerchiefs - a common activity in daily life. We expected that the sampling bias would be larger when the 
participants had to choose the handkerchief that they liked the most than when they had to choose the one they disliked the most, in accordance with the visual-preference and musicalpreference task literature discussed above. The present study also analyzed the relation between the sampling bias and task difficulty (i.e., the difference in preference level) in order to investigate the role of the sampling bias in the decisionmaking process. In previous studies, the gaze bias in a visual preference task was larger for the more difficult task (i.e., the difference in preference level was small; Shimojo et al., 2003). This indicates that the gaze bias is not merely a tendency to look at the chosen item, but rather is related to the decisionmaking process. Thus, we expected that the sampling bias in the haptic preference task would be larger for the more difficult task.

\section{Experiment 1}

Method

Participants Twenty-eight male students at Ritsumeikan University aged $21-25$ years $(M=21.9, S D=1.3)$ participated voluntarily in the experiment. This experiment was approved by the local ethics committee.

Materials Thirty handkerchiefs that differed in texture or weave were used for the experiment. Two handkerchiefs were presented $30 \mathrm{~cm}$ apart inside a box in front of the participants. The handkerchiefs were covered by the box, so the participants could not see them. The handkerchiefs differed in tactility. They were made of cotton, silk, hemp, artificial fabrics, terrycloth, etc. Some of them had embroidery, which provided a different tactile sensation.

Procedure We monitored hand movements when the participants were presented with a pair of handkerchiefs and asked them to identify the one they preferred the most or least. The participants were instructed to touch the handkerchiefs one at a time with their dominant hand. The experimenter told the participants which handkerchief to touch first at the beginning of each trial (i.e., "touch the right handkerchief first," or "touch the left handkerchief first") in an alternating order. The participants were instructed to put their dominant hand between the handkerchiefs and to report orally which handkerchief they liked more (the like task) or disliked more (the dislike task) as soon as they had made a decision. The instruction was made orally in Japanese ("Sukina hou wo erande kudasai" for the like task and "Kiraina hou wo erande kudasai" for the dislike task). After making their decision, the participants were instructed to rate the feel of the handkerchief on a 5-point scale $(1=$ very unfavorable, $2=$ unfavorable, $3=$ neutral, $4=$ favorable, and $5=$ very favorable).
The participants performed 15 trials in the like task and 15 trials in the dislike task on separate days, and task order was counterbalanced across participants. The entire experiment was completed in approximately $50 \mathrm{~min}$ each day. The 30 handkerchiefs were paired randomly for each participant and task. The participants did not judge the same handkerchief more than once in either task. Their behavior was recorded by a video camera. The beginning and end of a trial was determined by watching the videos. The time resolution of the video was $0.1 \mathrm{~s}$. We defined the beginning of a trial as the time of the first touch and the end as the time when the dominant hand was placed between the handkerchiefs. Repeatedly touching the same handkerchief was regarded as one sample when determining the amount of sampling per handkerchief.

\section{Results}

Table 1 shows a summary of the results. Effect sizes were estimated using Cohen's $d$ and $d_{z}$ for the standardized mean difference of an effect for between-subjects and withinsubjects designs, respectively (Lakens, 2013). The likelihood of sampling the chosen item last $(M=0.62)$ differed from chance $(0.5)$ in the like task, $t(27)=3.91, p<.001, d_{z}=0.74$, but not in the dislike task $(M=.49), t(27)=0.59, p=.56, d_{z}=$ 0.11 . The likelihood was also significantly larger in the like task than in the dislike task, $t(27)=3.99, p<.001, d_{z}=0.75, d$ $=0.94$, consistent with the results of previous studies involving visual (Mitsuda \& Glaholt, 2014; Schotter et al., 2010; Shimojo et al., 2003) and auditory (Lindsen et al., 2011) preference tasks. The likelihood of choosing the item sampled first did not differ significantly from chance for the like, $M=.50, t(27)=0, d_{z}=0$ or dislike, $M=.53, t(27)=1.20$, $p=.24, d_{z}=0.23$, tasks. Neither decision time (i.e., time until the end of the trial), $M=9.69 \mathrm{~s}$ and $10.83 \mathrm{~s}$ for the like and dislike tasks, respectively, $t(27)=1.39, p=.18, d_{z}=0.26$, $d=0.21$, nor the number of samples, $M=3.01$ and 3.06 for the like and dislike tasks, respectively, $t(27)=0.41, p=.69$, $d_{z}=0.08, d=0.06$ differed significantly between decision types (i.e., most versus least preferred). However, each participant's average preference rating across items was significantly larger in the like $(M=3.15)$ than the dislike $(M=3.01)$ task, $t(27)=2.62, p<.05, d_{z}=0.50, d=0.44$. Average preference ratings for each item across participants were also significantly larger in the like $(M=3.16)$ than the dislike $(M=3.03)$ task, $t(29)=3.90, p<.001, d_{z}=0.71, d=0.23$.

To investigate the relationship between task difficulty and sampling bias, we analyzed the difference in preference ratings between paired items (DP). Figure 1 shows the likelihood of sampling the chosen item last by DP. Because there were a small number of trials, DP3 and DP4 trials (i.e., those with 
Table 1 Summary of the results of Experiment 1. Values in parentheses indicate standard error of the mean

\begin{tabular}{lll}
\hline & Like task & Dislike task \\
\hline Likelihood of sampling the chosen item last & $0.62(0.03)$ & $0.49(0.02)$ \\
Likelihood of choosing the item sampled first & $0.50(0.02)$ & $0.53(0.03)$ \\
Decision time (s) & $9.69(0.84)$ & $10.83(1.21)$ \\
Number of samples & $3.01(0.15)$ & $3.06(0.17)$ \\
Average preference rating & $3.16(0.11)$ & $3.03(0.10)$ \\
\hline
\end{tabular}

difference scores of 3 or 4) were excluded from the analysis (there were $31[7.4 \%]$ and 40 [9.5\%] trials in the like and dislike tasks, respectively).

Shimojo et al. (2003) showed that gaze bias was larger when the task was more difficult. In accordance with their results, in the like task, bias was significantly larger for DP1 trials $(M=.71)$ than DP2 trials $(M=.55), t(27)=2.52, p<.05$, $d_{z}=0.48, d=0.29$. However, bias was significantly smaller for DP0 trials $(M=.46)$ than DP1 trials, $t(17)=2.69, p<.05$, $d_{z}=0.64, d=0.82$. The bias was significantly different from chance level for DP1 trials, $t(27)=5.99, p<.0001, d_{z}=1.1$, but not for DP2, $t(27)=0.95, p=.35, d_{z}=0.18$, or DP0 trials, $t(17)=0.50, p=.62, d_{z}=0.12$. The participants took significantly longer to make a choice, $t(17)=2.37, p<.05, d_{z}=$ $0.56, d=0.38$, on DP0 $(M=12.25 \mathrm{~s})$ than DP1 trials $(M=$ $10.32 \mathrm{~s})$. They also sampled significantly more items, $t(17)=$ $2.78, p<.05, d_{z}=0.66, d=0.63$, on DP0 $(M=3.66)$ than DP1 trials $(M=3.27)$. These results show that the participants made an effort to determine their preference between the two handkerchiefs on DP0 trials. Therefore, the low sampling bias on DP0 trials was not due to irregular behaviors, such as abandonment of the task. However, the bias on the dislike task did not reach significance on DP0, $M=.56, t(12)=0.61, p=.55$, $d_{z}=0.17, \mathrm{DP} 1, M=.46, t(27)=1.07, p=.30, d_{z}=0.20$, or DP2 trials, $M=.51, t(26)=0.21, p=.84, d_{z}=0.04$. Note that the difference in degrees of freedom in this latter analysis is due to the different number of available participants. One limitation of this analysis is the small number of samples on DP0 trials because ten participants did not rate any pair of items as equally preferable (DP0). Experiment 2 was designed to precisely investigate the sampling bias on DP0 trials.

\section{Experiment 2}

\section{Method}

Participants and materials Eighteen male students at Ritsumeikan University aged 20-23 years $(M=21.4, S D=$ 1.0 ) and who were not involved in Experiment 1 participated and received 500 JPY (approximately \$5 USD) for their participation. This experiment was approved by the local

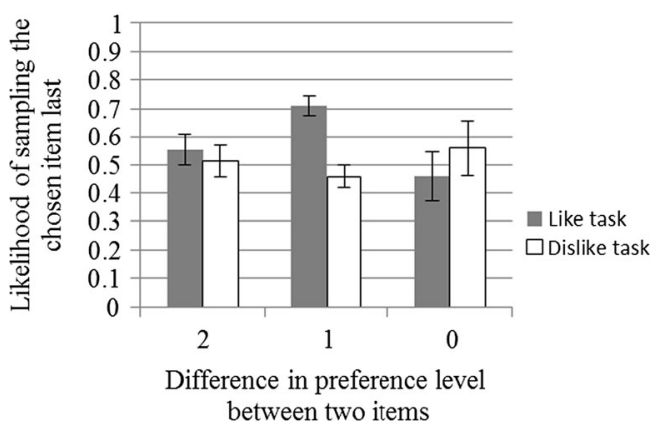

Fig. 1 The likelihood of sampling the chosen item last by the difference in preference level between two items. Error bars represent \pm 1 standard error of the mean

ethics committee. A further 15 types of handkerchiefs were added to the 30 types of handkerchiefs used in Experiment 1. In total, 45 types of handkerchiefs were used in Experiment 2. Pairs of different handkerchiefs were created by random selection from among 30 types of handkerchiefs. The remaining handkerchiefs (15 types) were used to create pairs of the same handkerchiefs.

Procedure In order to increase the number of trials in which participants rated two handkerchiefs as equally preferred, pairs of identical handkerchiefs were added to the task without notifying the participants. Thus, each task involved 15 pairs of two different handkerchiefs and 15 pairs of two identical handkerchiefs. The two types of pairs were presented randomly, and the handkerchiefs were randomly paired for each block of recording. The participants performed 30 trials for each like and dislike task on the same day. Then, the participants performed 30 trials from each task on an additional day, resulting in a total of 60 trials for each task. The order in which the like and dislike tasks were presented was counterbalanced across participants. The entire experiment was completed in approximately $50 \mathrm{~min}$ each day.

Hand movements were recorded by a magnetic position sensor (Patriot, Polhemus, Colchester, VT) at $60 \mathrm{~Hz}$ and by a video camera. We defined the duration of touching a handkerchief as the duration in which the hand was in either area of the box (i.e., the left or right side). The duration in which the hand was in the central area (i.e., the $10-\mathrm{cm}$ area between the handkerchiefs) was truncated so that it did not include the hand-movement time.

The task itself was the same as in Experiment 1 except that the participants were asked to press a key using their nondominant hand when making a decision (in Experiment 1, they placed their dominant hand between the handkerchiefs). The participants were instructed to press the " $X$ " key to select the right handkerchief and the " $\mathrm{Z}$ " key to select the left handkerchief. They were also asked to rate the handkerchiefs on each trial by pressing a button after recording their decision without touching the handkerchiefs again. 


\section{Results}

When the two handkerchiefs were identical, the participants reported a difference in preference (i.e., DP1 or DP2) on $39 \%$ of the trials $(210 / 540)$. This result shows that the participants were unable to discriminate the pairs of different handkerchiefs from the pairs of identical handkerchiefs. One possible explanation for the participants reporting different preference levels for two identical items is that the participants' touching behavior may have differed slightly between the two items because the items and their hand were not visible. Another possible explanation is that internal noise related to perception or a general prejudice that there were two different items may have resulted in differences in preference even when the touching behaviors were identical between the two items. Therefore, we analyzed their behavior without dissociating the two types of handkerchief pairs.

Table 2 shows a summary of the results. The likelihood of sampling the chosen item last differed from chance in the like $(M=.58), t(17)=3.59, p<.01, d_{z}=0.85$, but not the dislike $(M=.48)$ task, $t(17)=1.06, p=.30, d_{z}=0.25$, as in Experiment 1. The likelihood of choosing the item sampled first did not differ significantly from chance $(0.5)$ in the like $(M=.50), t(17)<0.01, d_{z}<0.01$, or dislike $(M=$ $.52)$ task, $t(17)=1.0, p=.33, d_{z}=0.24$. Neither decision time, $M=10.90 \mathrm{~s}$ and $11.31 \mathrm{~s}$ for the like and dislike tasks, respectively, $t(17)=0.97, p=.34, d_{z}=0.23, d=0.07$, nor the number of samples, $M=9.69 \mathrm{~s}$ and $10.83 \mathrm{~s}$ for the like and dislike tasks, respectively, $t(17)=0.06, d_{z}=0.01, d<$ 0.01 , differed significantly between decision types (i.e., preferred the most or least). Each participant's average preference rating across items was larger in the like $(M=$ 3.06) than the dislike $(M=3.00)$ task, $t(17)=1.99, p=.06$, $d_{z}=0.50, d=0.44$, although this finding did not reach statistical significance. The average preference ratings for each item across participants were significantly larger in the like task $(M=3.06)$ than the dislike $(M=3.00)$ task, $t(44)=2.99, p<.01, d_{z}=0.45, d=0.19$. All of these results are consistent with those of Experiment 1.

Figure 2 shows the decision time, number of samples, and the likelihood of sampling the chosen item last by DP. Because there were a small number of trials, DP3 and DP4 trials (39 trials [3.6\%] in the like task and 46 trials [4.3\%] in the dislike task) were excluded from the analysis. A $2 \times 3$ repeated measures ANOVA with task (like vs dislike) and difference in preference level (DP2, DP1, and DP0) as variables indicated no difference in decision time between the like and dislike tasks, $F(1,17)=0.82, p=.38, \eta_{\mathrm{p}}{ }^{2}=0.046$, or between the number of samples across the two tasks, $F(1,17)=0.001, p=$ $.97, \eta_{\mathrm{p}}{ }^{2}<0.01$. There were main effects of DP in the decision time, $F(2,34)=8.56, \eta_{\mathrm{p}}{ }^{2}=0.34, p<.001$, and in the number of samples, $F(2,34)=15.69, p<.001, \eta_{\mathrm{p}}{ }^{2}=0.48$. As in Experiment 1, the decision time was longer, and there were
Table 2 Summary of results of Experiment 2. Values in parentheses indicate standard error of the mean

\begin{tabular}{lll}
\hline & Like task & Dislike task \\
\hline Likelihood of sampling the chosen item last & $0.58(0.02)$ & $0.48(0.02)$ \\
Likelihood of choosing the item sampled first & $0.50(0.02)$ & $0.52(0.02)$ \\
Decision time (s) & $10.90(1.40)$ & $11.31(1.35)$ \\
Number of samples & $2.86(0.18)$ & $2.86(0.22)$ \\
Average preference rating & $3.06(0.07)$ & $3.00(0.07)$
\end{tabular}

larger samples, for smaller DP trials, indicating that the task was more difficult for smaller DPs.

The sampling bias in the like task was significantly greater than chance for DP1, $M=.61, t(17)=3.24, p<.01, d_{z}=0.76$, and DP0 trials, $M=.55, t(17)=2.18, p<.05, d_{z}=0.51$, but not for DP2 trials, $M=.47, t(17)=0.58, p=.57, d_{z}=0.14$. The sampling bias for DP0 trials $(0.55)$ was increased relative to that in Experiment 1 (0.46). However, it was smaller than that for DP1 trials (0.61), and the difference between DP1 trials and DP0 trials did not reach significance, $t(17)=1.37, p=.19$, $d_{z}=0.32$. The sampling bias in the dislike task did not differ from chance for DP2, $M=.51, t(17)=0.11, p=.91, d_{z}=0.03$, or DP1 trials, $M=.49, t(17)=0.28, p=.78, d_{z}=0.07$, which is consistent with the results of Experiment 1 . However, the bias on DP0 trials was significantly lower than chance, $M=.42$, $t(17)=2.61, p<.05, d_{z}=0.62$.

Finally, we analyzed the time course of sampling handkerchiefs. Previous studies on visual-preference judgments have shown that the duration of looking at an image just before a preference decision is significantly longer when the participants looked at the chosen image last compared to when they looked at the other image last (Glaholt \& Reingold, 2009; Mitsuda \& Glaholt, 2014; Schotter et al., 2010). This difference in gaze duration between the chosen and other image is regarded as an important factor in understanding the gaze bias.

We standardized the sampling duration to the mean of a single sampling duration for each participant in order to eliminate individual differences. Table 3 shows a summary of the results. The total sampling duration for the chosen item $(M=$ 1.45 and 1.40 for the like and dislike tasks, respectively) was significantly longer than that for the non-chosen item for the like task, $M=1.37, t(17)=2.83, p<.05, d_{z}=0.67, d=0.23$, but not for the dislike task, $M=1.44, t(17)=1.50, p=0.15, d_{z}$ $=0.35, d=0.08$. However, the mean sampling duration for the like task (i.e., the mean duration of one sampling) did not differ significantly between the chosen item $(M=1.01)$ and the other item $(M=1.01), t(17)<0.1, d_{z}=0.01, d=0.03$. Therefore, the longer total-sampling duration for the chosen item in the like task represents the effect of the greater number of samplings of the chosen item. The final sampling duration when participants sampled the chosen item $(M=0.90)$ was larger than the final sampling duration when participants 


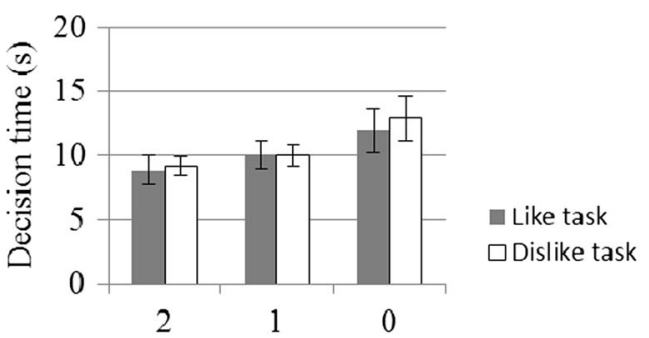

Difference in preference level between two items

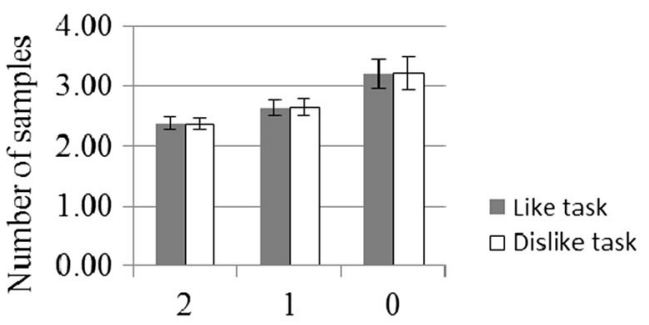

Difference in preference level between two items

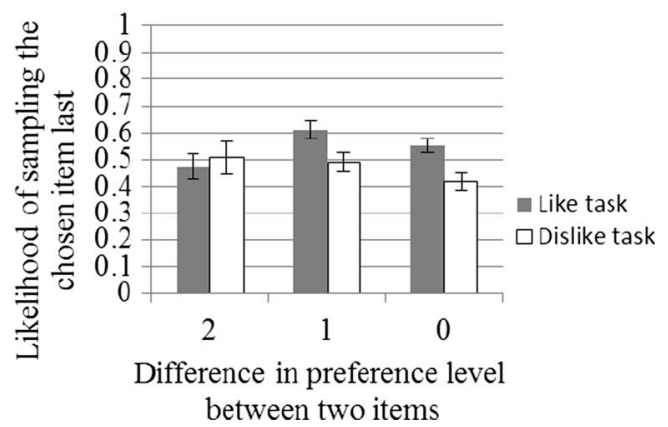

Fig. 2 Decision time, number of samples, and likelihood of sampling the chosen item last by difference in preference level for the two items. Error bars represent \pm 1 standard error of the mean

sampled the non-chosen item $(M=0.85)$ for the like task, $t(17)$ $=2.11, p=.05, d_{z}=0.50, d=0.30$, which is consistent with previous findings for a visual preference task (Mitsuda \& Glaholt, 2014). However, for the dislike task, the final sampling duration did not differ significantly between the chosen $(M=0.86)$ and non-chosen item $(M=0.89), t(17)=1.63, p=$ $.12, d_{z}=0.38, d=0.22$, which is inconsistent with previous

Table 3 Summary of standardized sampling durations. $100 *$ ratio to the mean sampling duration; values in parentheses indicate standard error of the mean

\begin{tabular}{llllll}
\hline & \multicolumn{2}{l}{ Like task } & & \multicolumn{2}{l}{ Dislike task } \\
\cline { 2 - 3 } \cline { 5 - 6 } \cline { 5 - 6 } & Chosen & Not-chosen & & Chosen & Not-chosen \\
\hline Total sampling duration & $145(10)$ & $137(8)$ & & $140(11)$ & $144(12)$ \\
Mean sampling duration & $101(1)$ & $101(1)$ & & $100(1)$ & $102(1)$ \\
First sampling duration & $108(3)$ & $107(2)$ & & $106(1)$ & $107(2)$ \\
Final sampling duration & $90(4)$ & $85(4)$ & & $86(4)$ & $89(4)$ \\
\hline
\end{tabular}

findings (Mitsuda \& Glaholt, 2014). The final sampling duration was significantly shorter than the mean sampling duration for both tasks (like task: $M=0.88, t(17)=3.11, p<.01, d_{z}=$ 0.73 ; dislike task: $\left.M=0.87, t(17)=3.70, p<.01, d_{z}=0.87\right)$, which is also consistent with previous findings (Mitsuda \& Glaholt, 2014). The first sampling duration did not differ significantly when the participants sampled the chosen (like task: $M=1.08$, dislike task: $M=1.07$ ) or non-chosen item first (like task: $M=1.06$, dislike task: $M=1.07)$ for the like, $t(17)=$ $0.50, p=.62, d_{z}=0.12, d=0.13$, or dislike tasks, $t(17)=1.04$, $p=.31, d_{z}=0.25, d=0.25$.

\section{Discussion}

The aim of this study was to test whether there is a sampling bias evident in haptic preference tasks that is similar to that observed in visual preference tasks. Experimental results showed that the participants tended to touch the handkerchief that they chose at the time in which they made their decision when they were asked to choose their preferred handkerchief, which is consistent with the results of a preference task involving music excerpts (Lindsen et al., 2011). These results indicate that the sampling bias in a preference-decision task is not specific to the visual modality; in other words, it is a general phenomenon, regardless of the sensory modality.

The likelihood of sampling the chosen item last ( 0.62 in Experiment 1 and 0.58 in Experiment 2) was smaller compared to that found in previous studies employing visual (Glaholt \& Reingold, 2009, 0.81; Schotter et al., 2010, 0.75; Shimojo et al., 2003, 0.83) or auditory (Lindsen et al., 2011, $0.73)$ preference tasks. These differences might be due to the greater effort required to sample items in the haptic (i.e., moving a hand from side to side) than in the visual (i.e., moving eyes) or auditory (i.e., pushing a button to change music) tasks. More effortful sampling can also explain why the sampling bias was smaller in the auditory preference task than in the visual preference tasks.

The difference in the sampling bias between this study and previous studies was greater in the dislike task. In the previous studies, the gaze bias in the dislike task was 0.56 (Shimojo et al., 2003) or 0.64 (Schotter et al., 2010), which was smaller than that in the like task but greater than chance. In contrast, in this study, the sampling bias in the dislike task was 0.49 and 0.48 in Experiments 1 and 2, respectively, which are approximately at chance level. In contrast, the sampling bias in the auditory dislike task was below chance $(0.44$, Lindsen et al., 2011). These inconsistencies require further study in order to identify the source of the sampling bias in the dislike task.

Heterogeneity in the results of the dislike task was also observed in the preference ratings. The preference ratings for the like task were significantly larger than those for the dislike task, which is consistent with the results of auditory 
preference tasks (Lindsen et al., 2011). Previous studies have shown that logically equivalent decisions, such as like vs dislike decisions, produce differences in cognitive processing (Meloy \& Russo, 2004; Shafir, 1993; Yamagishi \& Miyamoto, 1996). The differences between the like and dislike tasks observed in this study might reflect different decision processes for the different decision types.

The results of this study also show that differences in preference level affect the sampling bias. The sampling bias for DP1 trials was significantly larger than that for DP2 trials in the like task, which is consistent with previous results from a visual preference task (Shimojo et al., 2003). However this study also showed that the sampling bias for DP0 trials was smaller than that for DP1 trials. As described previously, the participants had to compare the handkerchiefs that were associated with similar tactile sensations or two identical handkerchiefs on many trials. Therefore, the DP0 trials in this study might have been more difficult than the trials categorized as difficult in the previous study (Shimojo et al., 2003). On DP0 trials, the participants had little or no differences in preference between the two items. Therefore, they might have chosen an item without forming a preference (i.e., no difference in preference level) despite there being a longer decision time than on DP1 trials. This means that the sampling bias is not related to task difficulty but to preference formation, as Shimojo et al. (2003) claimed.

There is a possibility that the sampling bias is not specific to preference formation. Several studies have shown that the gaze bias also exists in visual tasks that are not related to preference judgment. Glaholt and Reingold $(2009,2011)$ claimed that the gaze bias reflects the selective encoding of task-relevant information. The smaller sampling bias on DP0 trials can also be explained by this claim. In other words, the participant might not have been able to find the task-relevant information (i.e., preference) on DP0 trials despite the longer decision time. Investigating the sampling bias for haptic judgment tasks that are unrelated to preference is required to verify these claims.

This study also showed that the sampling bias for DP0 trials differed significantly from chance levels in the like task, though the magnitude of the biases were small. Contrary to expectations, the sampling bias for DP0 trials in the dislike task was smaller than chance levels. These results mean that, when the difference in preference level between two items was small, the participants tended to choose the item that they were touching in the like task and the other item in the dislike task. It is known that sampling order affects choice (Mantonakis et al., 2009). For DP0 trials, a recency effect (i.e., the most recently sampled item is recalled better) might result in greater preference for the item just sampled. Another possible source of the bias is the Mere Exposure Effect (Bornstein, 1989; Zajonc, 1968). Recently, the Mere Exposure Effect was demonstrated in the haptic modality (Jakesch \& Carbon, 2012). It has also been reported that consumers' evaluations of items increase when they are allowed to touch the items (Grohmann, Spagenberg, \&
Sprott, 2007; Peck \& Childers, 2003). The last item sampled tends to have a larger number of touches than the other items. Therefore, the Mere Exposure Effect might result in greater preference for the item sampled last. To dissociate the recency effect from the Mere Exposure Effect in DP0 trials, the number of touches on a trial and the number of items in the selection array (i.e., a multi-alternative forced-choice task) must be increased in future studies.

This study also analyzed the time course of sampling duration. The shorter duration for the final sampling compared to preceding samplings is consistent with the results from visual preference tasks (Mitsuda \& Glaholt, 2014). However, a shorter duration for the first sampling and a difference in the mean sampling duration between the chosen item and the other item was not observed in this study (although it has been observed in the visual preference tasks; Mitsuda \& Glaholt, 2014). These results show a significant difference in the time course of sampling duration between the eye movements and touching with a hand. The sampling duration analyzed in this study cannot distinguish between the duration of haptic inspection (i.e., perceiving the handkerchiefs) and the duration of determining preference (i.e., keeping a hand on a handkerchief). Further studies are required to dissociate these two behaviors.

The final sampling duration for the like task was significantly longer when the participants sampled the chosen item last than when they sampled the other item last, which is consistent with previous studies using a visual preference task (Glaholt \& Reingold, 2009; Mitsuda \& Glaholt, 2014). However, a bias in the final sampling duration for the dislike task was not observed in this study, although it was in a previous study (Mitsuda \& Glaholt, 2014). This might show that there is a relation between a bias in the final sampling duration and a bias in the likelihood of sampling the chosen item last because the likelihood of sampling the chosen item last was significantly greater than chance in the previous study but not in this study.

In summary, the present study demonstrated the sampling bias in a haptic preference task, which indicates that the sampling bias is a general phenomenon regardless of sensory modality. In addition, this study showed that the increase in sampling bias is related to preference formation and/or the selective encoding of task-relevant information as well as task difficulty. This indicates that records of sampling behaviors in preference-judgment tasks have the potential to increase our understanding of the decision-making process.

\section{References}

Bornstein, R. F. (1989). Exposure and affect: Overview and meta-analysis of research, 1968-1987. Psychological Bulletin, 106, 265-289. doi: 10.1037/0033-2909.106.2.265 
Glaholt, M. G., \& Reingold, E. M. (2009). The time course of gaze bias in visual decision tasks. Visual Cognition, 17, 1228-1243. doi:10. 1080/13506280802362962

Glaholt, M. G., \& Reingold, E. M. (2011). Eye movement monitoring as a process tracing methodology in decision making research. Journal of Neuroscience, Psychology, and Economics, 4, 125-146. doi:10. 1037/a0020692

Grohmann, B., Spagenberg, E. R., \& Sprott, D. E. (2007). The influence of tactile input on the evaluation of retail product offerings. Journal of Retailing, 83, 237-245. doi:10.1016/j.jretai.2006.09.001

Jakesch, M., \& Carbon, C. C. (2012). The mere exposure effect in the domain of haptics. PloS One, 7, e31215. doi:10.1371/journal.pone. 0031215

Krajbich, I., Armel, C., \& Rangel, A. (2010). Visual fixations and the computation and comparison of value in simple choice. Nature Neuroscience, 13, 1292-1298. doi:10.1038/nn.2635

Lakens, D. (2013). Calculating and reporting effect sizes to facilitate cumulative science: A practical primer for t-tests and ANOVAs. Frontiers in Psychology, 4, 1-12. doi:10.3389/fpsyg.2013. 00863

Lindsen, J. P., Gurpreet, M., Shimojo, S., \& Bhattacharya, J. (2011). Swayed by the music: Sampling bias towards musical preference distinguishes like from dislike decisions. Consciousness and Cognition, 20, 1781-1786. doi:10.1016/j.concog.2011.01.008

Mantonakis, A., Rodero, P., Lesschaeve, I., \& Hastie, R. (2009). Order in choice: Effects of serial position on preferences. Psychological Science, 20, 1309-1312. doi:10.1111/j.1467-9280.2009.02453.x
Meloy, M. G., \& Russo, J. E. (2004). Binary choice under instructions to select versus reject. Organizational Behavior and Human Decision Processes, 93, 114-128. doi:10.1016/j.obhdp.2003.12.002

Mitsuda, T., \& Glaholt, M. G. (2014). Gaze bias during visual preference judgments: Effects of stimulus category and decision instructions. Visual Cognition, 22, 1-29. doi:10.1080/13506285.2014.881447

Nittono, H., \& Wada, Y. (2009). Gaze shifts do not affect preference judgments of graphic patterns. Perceptual and Motor Skills, 109, 79-94. doi:10.2466/pms. 109.1.79-94

Peck, J., \& Childers, T. L. (2003). To have and to hold: The influence of haptic information on product judgments. Journal of Marketing, 67, 35-48. doi:10.1509/jmkg.67.2.35.18612

Schotter, E. R., Berry, R. W., McKenzie, C. R. M., \& Rayner, K. (2010). Gaze bias: Selective encoding and liking effects. Visual Cognition, 18, 1113-1132. doi:10.1080/13506281003668900

Shafir, E. (1993). Choosing versus rejecting: Why some options are both better and worse than others. Memory \& Cognition, 21, 546-556. doi:10.3758/BF03197186

Shimojo, S., Simion, C., Shimojo, E., \& Scheier, C. (2003). Gaze bias both reflects and influences preference. Nature Neuroscience, 6 , 1317-1322. doi:10.1038/nn1150

Yamagishi, K., \& Miyamoto, J. M. (1996). Asymmetries in strength of preference: A focus shift model of valence effects in difference judgments. Journal of Experimental Psychology: Learning, Memory, and Cognition, 22, 493-509. doi:10.1037/0278-7393.22.2.493

Zajonc, R. B. (1968). Attitudinal effects of mere exposure. Journal of Personality and Social Psychology, 9, 1-27. doi:10.1037/h0025848 